\title{
Upper Domination: Complexity and Approximation
}

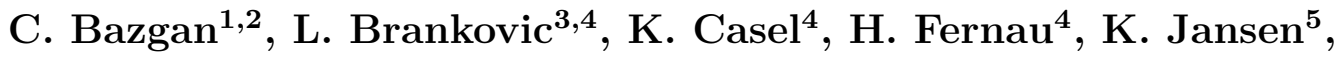 \\ K. Klein ${ }^{5}$, M. Lampis ${ }^{1}$, M. Liedloff ${ }^{6}$, J. Monnot ${ }^{1}$, and V. Paschos ${ }^{1}$ \\ 1 PSL, University of Paris-Dauphine, CNRS, LAMSADE UMR 7243, \\ F-75775 Paris Cedex 16, France \\ \{bazgan, michail.lampis, jerome.monnot, paschos\}@lamsade.dauphine.fr \\ 2 Institut Universitaire de France \\ 3 The University of Newcastle, Callaghan, NSW 2308, Australia \\ ljiljana.brankovic@newcastle.edu.au \\ 4 Universität Trier, Fachbereich 4, Informatikwissenschaften, \\ D-54286 Trier, Germany, \{casel, fernau\}@uni-trier.de \\ 5 Universität Kiel, Institut für Informatik, D-24098 Kiel, Germany \\ $\{k j, k m k\} @ i n f o r m a t i k$.uni-kiel.de \\ 6 Univ. Orléans, INSA Centre Val de Loire, LIFO EA 4022, \\ F-45067 Orléans, France, mathieu.liedloff@univ-orleans.fr
}

\begin{abstract}
We consider the UPPER Domination problem, which is the problem of finding a maximum cardinality minimal dominating set in a graph. We show that this problem does not admit an $n^{1-\epsilon}$ approximation for any $\epsilon>0$, making it significantly harder than Dominating SET, while it remains hard even on severely restricted special cases, such as cubic graphs (APX-hard), and planar subcubic graphs (NP-hard). One of our main contributions is to pinpoint an exact reason why this variant of Dominating SET is harder: we show that in general, when given a set of vertices $S$, deciding if a minimal dominating set of any size may contain $S$ is NP-hard. Being able to decide such extension questions, which are trivial for InDEPENDENT SET and most other maximisation problems, is a prerequisite for running most natural approximation algorithms.

We complement our negative results by showing that the problem admits an $O(\Delta)$ approximation on graphs of maximum degree $\Delta$, as well as an EPTAS on planar graphs. Along the way, we also derive essentially tight $n^{1-\frac{1}{d}}$ upper and lower bounds on the approximability of the related Maximum Minimal Hitting SeT problem on $d$-uniform hypergraphs, generalising corresponding known results for Maximum Minimal Vertex Cover.
\end{abstract}

\section{Introduction}

A dominating set of an undirected graph $G(V, E)$ is a set of vertices $S \subseteq V$ such that all vertices outside of $S$ have a neighbour in $S$. The problem of finding the smallest dominating set of a given graph is one of the most intensively studied problems in computational complexity. In this paper, we focus on a related problem that "flips" the optimisation objective. In UPPER Domination we are given a graph and we are asked to find a maximum cardinality dominating set that is still minimal. A dominating set is minimal if any proper subset of it is no longer dominating, that is, if it does not contain obviously redundant vertices.

Considering a MaxMin or MinMax version of a problem by "flipping" the objective is not a new idea; in fact, such questions have been posed before for many classical optimisation problems. Some of the most well-known examples include the Minimum Maximal IndePendent Set problem $[13,9,8,18$ ] (also known as Minimum Independent Dominating Set), the Maximum Minimal Vertex Cover problem $[7,23]$ and the Lazy BureauCRAT problem [2, 4], which is a MinMax version of KNAPSACK. The initial motivation for this type of question was rather straightforward: most classical optimisation problems admit

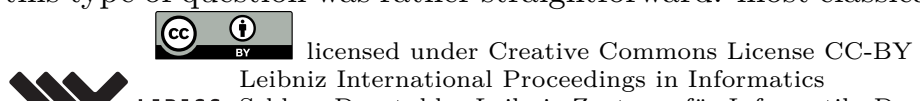


an easy, naive heuristic algorithm which starts with a trivial solution and then gradually tries to improve it in an obvious way until it gets stuck. For example, one can produce a (maximal) independent set of a graph by starting with a single vertex and then adding vertices to the current solution while maintaining an independent set. What can we say about the worst-case performance of such a basic algorithm? Motivated by this initial question the study of MaxMin and MinMax versions of standard optimisation problems has gradually grown into a sub-field with its own interest, often revealing new insights on the structure of the original problems. UPPER DOMINATION is a natural example of this family of problems, on which somewhat fewer results are currently known. The goal of this paper is to increase our understanding of this problem and provide new algorithmic and complexity results.

A typical pattern that often shows up in this line of research is that MaxMin versions of classical problems turn out to be much harder than the originals, especially when one considers approximation. For example, Maximum Minimal Vertex Cover does not admit any $n^{\frac{1}{2}-\epsilon}$ approximation, while VERTEX COVER admits a 2-approximation [7]; LAZY Bureaucrat is APX-hard while Knapsack admits a PTAS [2]; and though Minimum Maximal Independent Set and Independent Set share the same (inapproximable) status, the proof of inapproximability of the MinMax version is considerably simpler, and was known long before corresponding hardness results for InDEPEndent SET [13].

Our first contribution in this paper is to show that this pattern also holds for UpPER Domination: while Dominating Set admits a greedy $\ln n$-approximation, UpPer DominATION does not admit an $n^{1-\epsilon}$ approximation for any $\epsilon>0$, unless $\mathrm{P}=\mathrm{NP}$. We establish this by considering the related Maximum Minimal Hitting SeT problem: given a $d$-uniform hypergraph, find the largest minimal set of vertices that intersects all hyperedges. Observe that the previously studied Maximum Minimal Vertex Cover problem is a special case of this problem for $d=2$. We show, for any $d$, an approximation algorithm with ratio $n^{1-\frac{1}{d}}$, for Maximum Minimal Hitting Set on $d$-uniform hypergraphs, as well as a tight $n^{1-\frac{1}{d}-\epsilon}$ inapproximability bound, exactly matching, and subsuming, the corresponding tight $\sqrt{n}$ approximation results for Maximum Minimal Vertex Cover given in [7]. We then obtain the inapproximability of UpPer Domination by performing a reduction from an instance with sufficiently large $d$. We also show that UPPER DOMINATION remains hard on two restricted cases: the problem is still APX-hard on cubic graphs, and NP-hard on planar subcubic graphs. Since the problem is easy on graphs of maximum degree 2, our results completely characterise the complexity of the problem in terms of maximum degree (the best previously known result was NP-hardness for planar graphs of maximum degree 6 [1]).

A question one may ask, given the general behavior of this type of problem, and the above results on UPPER DOMINATION in particular, is why are such problems typically so much harder than their original versions? What is perhaps the main conceptual contribution of this paper is to give a concrete and intuitive answer to this question. We consider the following extension problem: Given a graph $G(V, E)$ and a set $S \subseteq V$, does there exist a minimal dominating set of any size that contains $S$ ? Even though questions of this type are typically trivial for problems such as IndePEndent SET and Lazy Bureaucrat, we show that in this case, deciding the existence of such a minimal dominating set is NP-hard. We posit that this helps explain the added difficulty of this problem, and more generally of problems of this type, since any natural algorithm that gradually builds a solution would have to contend with (some version of) this extension problem.

We complement the above negative results by giving some approximation algorithms for the problem in restricted cases. Specifically, we show that the problem admits an $O(\Delta)$ approximation on graphs with maximum degree $\Delta$, as well as an EPTAS on planar graphs. 


\section{Previous results}

It has long been known that UPPER DOMination is NP-complete in general [10], and even for graphs of maximum degree 6 [1]. Some polynomial-time solvable graph classes are also known. This is mainly due to the fact that on certain graph classes (like bipartite graphs) the independence number and upper domination number coincide and for those graph classes, the independence number can be computed in polynomial-time. We refer to the textbook on domination [15] for further details. We mention that the problem is polynomial for bipartite graphs [11], chordal graphs [19] and generalised series-parallel graphs [14]. Very recently, the complexity of UPPER DOMINATION in monogenic classes of graphs, i.e. classes defined by a single forbidden induced subgraph, has led to a complexity dichotomy: if the unique forbidden induced subgraph is a $P_{4}$ or a $2 K_{2}$ (or an induced subgraph of these), then UPPER Domination can be solved in polynomial time; otherwise, it is NP-complete [1].

\section{Preliminaries}

We only deal with undirected simple graphs $G=(V, E)$. The number of vertices $n=|V|$ is known as the order of $G$. As usual, $N(v)$ denotes the open neighbourhood of $v$, and $N[v]$ is the closed neighbourhood of $v$, i.e., $N[v]=N(v) \cup\{v\}$. These notions can be easily extended to vertex sets $X$, i.e., $N(X)=\bigcup_{x \in X} N(x)$. The cardinality of $N(v)$ is also known as the degree of $v$, denoted as $\operatorname{deg}(v)$. The maximum degree in a graph is written as $\Delta$. A graph of maximum degree three is called subcubic, and if all degrees equal three, it is called cubic.

Given a graph $G=(V, E)$, a subset $S$ of $V$ is a dominating set if every vertex $v \in V \backslash S$ has at least one neighbour in $S$, i.e., if $N[S]=V$. A dominating set is minimal if no proper subset of it is a dominating set. Likewise, a vertex set $I$ is independent if $N(I) \cap I=\emptyset$. An independent set is maximal if no proper superset is independent. In the following we use classical notations: $\gamma(G)$ and $\Gamma(G)$ are the minimum and maximum cardinalities over all minimal dominating sets in $G, \alpha(G)$ is the maximum cardinality of an independent set, $i(G)$ is the minimum cardinality of a maximal independent set, and $\tau(G)$ is the size of a minimum vertex cover, which equals $|V|-\alpha(G)$ by Gallai's identity. A minimal dominating set $D$ of $G$ with $|D|=\Gamma(G)$ is also known as an upper dominating set of $G$.

For any subset $S \subseteq V$ and $v \in S$ we define the private neighbourhood of $v$ with respect to $S$ as $p n(v, S):=N[v]-N[S-\{v\}]$. Any $w \in p n(v, S)$ is called a private neighbour of $v$ with respect to $S$. If the set $S$ is clear from the context, we will omit the "with respect to $S$ " part. $S$ is called irredundant if every vertex in $S$ has at least one private neighbour, i.e., if $|p n(v, S)|>0$ for every $v \in S$. $\operatorname{IR}(G)$ denotes the cardinality of the largest irredundant set in $G$, while $\operatorname{ir}(G)$ is the cardinality of the smallest maximal irredundant set in $G$.

We can now observe the validity of the well-known domination chain.

$$
\operatorname{ir}(G) \leq \gamma(G) \leq i(G) \leq \alpha(G) \leq \Gamma(G) \leq \operatorname{IR}(G)
$$

The domination chain is largely due to the following two combinatorial properties: (1) Every maximal independent set is a minimal dominating set. (2) A dominating set $S \subseteq V$ is minimal if and only if $|p n(v, S)|>0$ for every $v \in S$. Observe that $v$ can be a private neighbour of itself, i.e., a dominating set is minimal if and only if it is also an irredundant set. Actually, every minimal dominating set is also a maximal irredundant set. 


\section{Notes on the combinatorial structure of minimal dominating sets}

Any minimal dominating set $D$ for a graph $G=(V, E)$ can be associated with a partition of the set of vertices $V$ into four sets $F, I, P, O$ given by: $I:=\{v \in D: v \in p n(v, D)\}$, $F:=D-I, P \in\{B \subseteq N(F) \cap(V-D):|p n(v, D) \cap B|=1$ for all $v \in F\}$ with $|F|=|P|$, $O=V-(D \cup P)$. This representation is not necessarily unique since there might be different choices for $P$ and $O$, but for every partition of this kind, the following properties hold:

1. Every vertex $v \in F$ has at least one neighbour in $F$, called a friend.

2. The set $I$ is an independent set in $G$.

3. The subgraph induced by the vertices $F \cup P$ has an edge cut set separating $F$ and $P$ that is, at the same time, a perfect matching; hence, $P$ can serve as the set of private neighbours for $F$.

4. The neighbourhood of a vertex in $I$ is always a subset of $O$, which are otherwise the outsiders.

This partition is also related to a different characterisation of $\Gamma(G)$ in terms of so-called upper perfect neighbourhoods [15].

- Lemma 1. For any connected graph $G$ with $n>0$ vertices and an upper dominating set $D$ with an associated partition $(F, I, P, O)$ as defined above, if $|D|=\Gamma(G)>\alpha(G)$ then $|I| \leq \alpha(G)-2$.

- Lemma 2. For any connected graph $G$ with $n>0$ vertices we have:

$$
\alpha(G) \leq \Gamma(G) \leq \max \left\{\alpha(G), \frac{n}{2}+\frac{\alpha(G)}{2}-1\right\}
$$

The following lemma generalises the earlier result on upper bounds on $\operatorname{IR}(G)$ (and hence on $\Gamma(G))$ for $\Delta$-regular graphs $G$, which is $\operatorname{IR}(G) \leq n / 2$; see [16, Proposition 12]. Notice that our result generalises the mentioned result of Henning and Slator, as minimum and maximum degrees coincide for regular graphs.

- Lemma 3. For any connected graph $G$ with $n>0$ vertices, minimum degree $\delta$ and maximum degree $\Delta$, we have:

$$
\alpha(G) \leq \Gamma(G) \leq \max \left\{\alpha(G), \frac{n}{2}+\frac{\alpha(G)(\Delta-\delta)}{2 \Delta}-\frac{\Delta-\delta}{\Delta}\right\}
$$

\section{$4 \quad$ Hardness Results for Upper Domination}

In this section we demonstrate several results that indicate that UPPER Domination is a rather hard problem: it does not admit any non-trivial approximation in polynomial time, and it remains hard even in quite restricted cases.

\subsection{Hardness of Approximation on General Graphs}

We show that Upper Domination is hard to approximate in two steps: first, we show that a related natural problem, Maximum Minimal Hitting Set, is hard to approximate, and then we show that this problem is essentially equivalent to UPPER Domination.

The Maximum Minimal Hitting Set problem is the following: we are given a hypergraph, that is, a base set $V$ and a collection $F$ of subsets of $V$. We wish to find a set $H \subseteq V$ such that: (1) For all $e \in F$ we have $e \cap H \neq \emptyset$ (i.e., $H$ is a hitting set) (2) For all $v \in H$ 
there exists $e \in F$ such that $e \cap H=\{v\}$ (i.e., $H$ is minimal) (3) $H$ is as large as possible. This problem generalises UpPeR Domination: given a graph $G=(V, E)$, we can produce a hypergraph by keeping the same set of vertices and creating a hyperedge for each closed neighbourhood $N[v]$ of $G$. An upper dominating set of the original graph is now exactly a minimal hitting set of the constructed hypergraph. We will also show that MAXIMUM Minimal Hitting Set can be reduced to Upper Domination.

Let us note that Maximum Minimal Hitting Set, as defined here, also generalises Maximum Minimal Vertex Cover, which corresponds to instances where the input hypergraph is actually a graph. We recall that for this problem there exists a $n^{1 / 2_{-}}$ approximation algorithm, while it is known to be $n^{1 / 2-\varepsilon}$-inapproximable [7]. Here, we generalise this result to arbitrary hypergraphs, taking into account the sizes of the hyperedges allowed.

- Theorem 4. For all $\varepsilon>0, d \geq 2$, if there exists a polynomial-time approximation algorithm for MAXimum Minimal Hitting SET which on hypergraphs $G=(V, F)$ where hyperedges have size exactly $d$ has approximation ratio $n^{\frac{d-1}{d}-\varepsilon}$, where $|V|=n$, then $P=N P$. This is still true for hypergraphs where $|F|=O(|V|)$.

Proof. Fix some constant hyperedge size $d$. We will present a reduction from MAXIMUM InDEPENDENT SET, which is known to be inapproximable [17]. Specifically, for all $\varepsilon>0$, it is known to be NP-hard to distinguish for an $n$-vertex graph $G$ if $\alpha(G)>n^{1-\varepsilon}$ or $\alpha(G)<n^{\varepsilon}$.

Take an instance $G=(V, E)$ of Maximum Independent Set. If $d>2$ we begin by turning $G$ into a $d$-uniform hypergraph $G^{\prime}=(V, H)$ such that any (non-trivial) hitting set of $G^{\prime}$ is a vertex cover of $G$ and vice-versa (for $d=2$ we simply set $G^{\prime}=G$ ). We proceed as follows: for every edge $e \in E$ and every $S \subseteq V \backslash e$ with $|S|=d-2$ we construct in $H$ the hyperedge $e \cup S$ (with size exactly $d$ ). Thus, $|H|=O\left(n^{d}\right)$. Any vertex cover of $G$ is also a hitting set of $G^{\prime}$. For the converse, we only want to prove that any hitting set of $G^{\prime}$ of size at most $n-d$ is also a vertex cover of $G$ (this is without loss of generality, since $d$ is a constant, so we will assume $\alpha(G)>d$ ). Take a hitting set $C$ of $G^{\prime}$ with at most $n-d$ vertices; take any edge $e \in E$ and a set $S$ with $S \subseteq V \backslash(C \cup e)$ and $|S|=d-2$ (such a set $S$ exists since $|V \backslash C| \geq d)$. Now, $(e \cup S) \in H$, therefore $C$ must contain a vertex of $e$. We thus conclude that the maximum size of $V \backslash C$, where $C$ is a hitting set of $G^{\prime}$ is either at least $n^{1-\varepsilon}$ or at most $n^{\varepsilon}$, that is, the maximum size of $V \backslash C$ is $\alpha(G)$.

We now add some vertices and hyperedges to $G^{\prime}$ to obtain a hypergraph $G^{\prime \prime}$. For every set $S \subseteq V$ such that $|S|=d-1$ and $V \backslash S$ is a hitting set of $G^{\prime}$, we add to $G^{\prime \prime} n$ new vertices, call them $u_{S, i}, 1 \leq i \leq n$. Also, for each such vertex $u_{S, i}$ we add to $G^{\prime \prime}$ the hyperedge $S \cup\left\{u_{S, i}\right\}, 1 \leq i \leq n$. This completes the construction. It is not hard to see that $G^{\prime \prime}$ has hyperedges of size exactly $d$, and its vertex and hyperedge set are both of size $O\left(n^{d}\right)$.

Let us analyse the approximability gap of this reduction. First, suppose that there is a minimal hitting set $C$ of $G^{\prime}$ with $|V \backslash C|>n^{1-\varepsilon}$. Then, there exists a minimal hitting set of $G^{\prime \prime}$ with size at least $n^{d-O(d \varepsilon)}$. To see this, consider the set $C \cup\left\{u_{S, i} \mid S \subseteq V \backslash C, 1 \leq i \leq n\right\}$. This set is a hitting set, since $C$ hits all the hyperedges of $G^{\prime}$, and for every new hyperedge of $G^{\prime \prime}$ that is not covered by $C$ we select $u_{S, i}$. It is also minimal, because $C$ is a minimal hitting set of $G^{\prime}$, and each $u_{S, i}$ selected has a private hyperedge. To calculate its size, observe that for each $S \subseteq V \backslash C$ with $|S|=d-1$ we have $n$ vertices. There are at least $\left(\begin{array}{c}n^{1-\varepsilon} \\ d-1\end{array}\right)$ such sets.

For the converse direction, we want to show that if $|V \backslash C|<n^{\varepsilon}$ for all hitting sets $C$ of $G^{\prime}$, then any minimal hitting set of $G^{\prime \prime}$ has size at most $n^{1+O(d \varepsilon)}$. Consider a hitting set $C^{\prime}$ of $G^{\prime \prime}$. Then, $C^{\prime} \cap V$ is a hitting set of $G^{\prime}$. Let $S \subset V$ be a set of vertices such that $S \cap C^{\prime} \neq \emptyset$. Then $u_{S, i} \notin C^{\prime}$ for all $i$, because the (unique) hyperedge that contains $u_{S, i}$ also contains 
some other vertex of $C^{\prime}$, contradicting minimality. Now, because $V \cap C^{\prime}$ is a hitting set of $G^{\prime}$ we have $\left|V \backslash C^{\prime}\right| \leq n^{\varepsilon}$. Thus, the maximum number of different sets $S \subseteq V$ such that some $u_{S, i} \in C^{\prime}$ is $\left(\begin{array}{c}n^{\varepsilon} \\ d-1\end{array}\right)$ and the total size of $C^{\prime}$ is at most $\left|C^{\prime} \cap V\right|+n^{\varepsilon(d-1)+1} \leq n^{1+O(d \varepsilon)}$.

- Corollary 5. For any $\varepsilon>0$ Maximum Minimal Hitting Set is not $n^{1-\varepsilon}$-approximable, where $n$ is the number of vertices of the input hypergraph, unless $P=N P$. This is still true for hypergraphs $G=(V, F)$ where $|F| \in O(|V|)$.

- Theorem 6. For any $\varepsilon>0$ UPPER DOMINATION is not $n^{1-\varepsilon}$-approximable, where $n$ is the number of vertices of the input graph, unless $P=N P$.

Note that, in fact, the inapproximability bound given in Theorem 4 is tight, for every fixed $d$, a fact that we believe may be of independent interest. This is shown in the following theorem, which also generalises results on Maximum Minimal Vertex Cover [7].

- Theorem 7. For all $d \geq 1$, there exists a polynomial-time algorithm which, given a hypergraph $G=(V, F)$ such that all hyperedges have size at most $d$, produces a minimal hitting set $H$ of $G$ with size $\Omega\left(n^{1 / d}\right)$. This shows an $\Omega\left(n^{\frac{d-1}{d}}\right)$-approximation for MAXIMUM Minimal Hitting SET on such hypergraphs.

\subsection{Hardness on Cubic and Subcubic Planar Graphs}

UpPeR Domination has been shown to be NP-hard on planar graphs of maximum degree six [1]. Here, we are going to strengthen this result in two directions: first, we show that even for cubic graphs the problem is APX-hard; second, the problem remains NP-hard for planar subcubic graphs. We later complement this hardness with an EPTAS on planar graphs.

- Theorem 8. UPPER Domination is APX-hard on cubic graphs.

Proof. (Sketch) We present a reduction from MAXIMum IndePEndent SeT on cubic graphs, which is known to be APX-hard [22]. Let $G=(V, E)$ be the cubic input graph.

Build $G^{\prime}$ from $G$ by replacing every $(u, v) \in E$ by a construction introducing six new vertices, as shown on the right. Any $I S \subset V$ is an independent set for $G$ if and only if $G^{\prime}$ contains an upper dominating set of cardinality $|I S|+3|E|$.

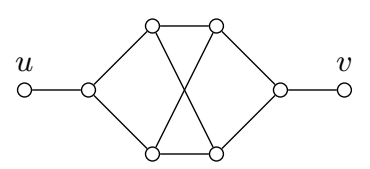

- Theorem 9. UPPER DOMINATION is NP-hard on planar subcubic graphs

\section{What makes this problem that hard?}

Algorithms working on combinatorial graph problems often try to look at local parts of the graph and then extend some part of the (final) solution that was found and fixed so far. This type of strategy is at least difficult to implement for UPPER DOMINATION, as the following example shows.

First, consider a graph $G_{n}$ that consists of two cliques with vertices $V_{n}=\left\{v_{1}, \ldots, v_{n}\right\}$ and $W_{n}=\left\{w_{1}, \ldots, w_{n}\right\}$, where the only edges connecting both cliques are $v_{i} w_{i}$ for $1 \leq i \leq n$. Observe that $G_{n}$ has as minimal dominating sets $V_{n}, W_{n}$, and $\left\{v_{i}, w_{j}\right\}$ for all $1 \leq i, j \leq n$. For $n \geq 3$, the first two are upper dominating sets, while the last $n^{2}$ many are minimum dominating sets. If we now add a vertex $v_{0}$ to $G_{n}$, arriving at graph $G_{n}^{\prime}$, and make $v_{0}$ adjacent to all vertices in $V_{n}$, then $V_{n}$ is still a minimal dominating set, but $W_{n}$ is no longer 


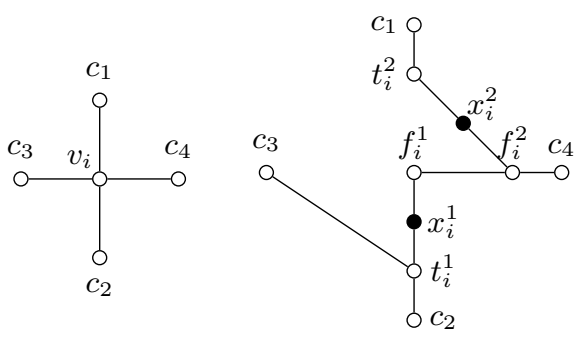

$v_{i} \in c_{1}, c_{2}, c_{3}, \bar{v}_{i} \in c_{4}$

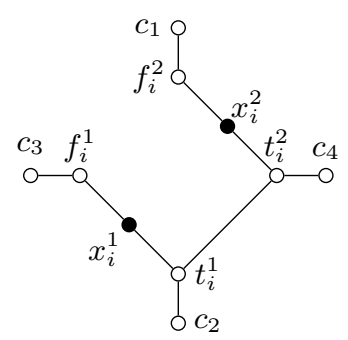

$v_{i} \in c_{2}, c_{4}, \bar{v}_{i} \in c_{1}, c_{3}$

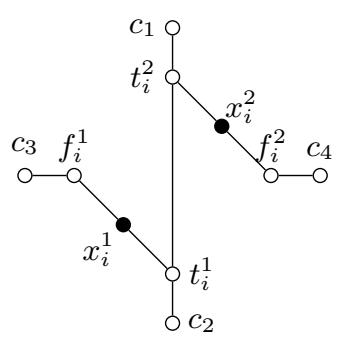

$v_{i} \in c_{1}, c_{2}, \bar{v}_{i} \in c_{3}, c_{4}$

Figure 1 Construction of Theorem 10: A variable $v_{i}$ appearing in four clauses $c_{1}, \ldots, c_{4}$, of the original instance is transformed to one of the subgraphs on the right, depending on which clauses it appears positive in. Black vertices denote elements of $S$.

a dominating set. Now, we have $\left\{v_{i}, w_{j}\right\}$ for all $0 \leq i \leq n$ and all $1 \leq j \leq n$ as minimum dominating sets. But, if we add one further vertex, $w_{0}$ to $G_{n}^{\prime}$ to obtain $G_{n}^{\prime \prime}$ and make $w_{0}$ adjacent to all vertices in $W_{n}$, then all upper dominating sets are also minimum dominating sets and vice versa. This shows that we cannot consider vertices one by one, but must rather look at the whole graph.

For many maximisation problems, like UpPeR IRREDUndance or MAXIMUM IndEPENDENT SET, it is trivial to obtain a feasible solution that extends a given vertex set by some greedy strategy, or to know that no such extension exists. This is not true for UpPER Domination, as we show next. Let us first define the problem formally.

Minimal Dominating Set Extension
Input: A graph $G=(V, E)$, a set $S \subseteq V$.
Question: Does $G$ have a minimal dominating set $S^{\prime}$ with $S^{\prime} \supseteq S$ ?

Notice that this problem is trivial on some input with $S=\emptyset$ by using a greedy approach. However, this strategy might fail if the given set $S$ is bigger, as we must also maintain the property of being superset of $S$. This difficulty is very hard to overcome, as the next result shows. Its proof is based on a reduction from the NP-complete 4-Bounded PLANAR 3-CONNECTED SAT problem (4P3C3SAT for short) [20], the restriction of 3-satisfiability to clauses in $C$ over variables in $V$, where each variable occurs in at most four clauses and the associated bipartite graph $(C \cup X,\{(c, x) \in C \times X:(x \in c) \vee(\neg x \in c)\})$ is planar.

- Theorem 10. Minimal Dominating Set Extension is NP-complete, even when restricted to planar cubic graphs.

Proof. Membership in NP is obvious. Reduction from 4P3C3SAT shows NP-hardness: Consider an instance of 4P3C3SAT with clauses $c_{1}, \ldots, c_{m}$ and variables $v_{1}, \ldots, v_{n}$. By definition, the graph $G=(V, E)$ with $V=\left\{c_{1}, \ldots, c_{m}\right\} \cup\left\{v_{1}, \ldots, v_{n}\right\}$ and $E=\left\{\left(c_{j}, v_{i}\right): v_{i}\right.$ or $\bar{v}_{i}$ is literal of $\left.c_{j}\right\}$ is planar. Replace every vertex $v_{i}$ by six new vertices $f_{i}^{1}, x_{i}^{1}, t_{i}^{1}, t_{i}^{2}, x_{i}^{2}, f_{i}^{2}$ with edges $\left(f_{i}^{j}, x_{i}^{j}\right),\left(t_{i}^{j}, x_{i}^{j}\right)$ for $j=1,2$. If $v_{i}$ (positive) is a literal in more than two clauses, add the edge $\left(f_{i}^{1}, f_{i}^{2}\right)$, else add the edge $\left(t_{i}^{1}, t_{i}^{2}\right)$. By definition of the problem 4P3C3SAT, each variable appears in at most four clauses and this procedure of replacing the variablevertices in $G$ by a $P_{6}$ preserves planarity. To see this, consider any fixed planar embedding of $G$ and any variable $v_{i}$ which appears in clauses $c_{1}, c_{2}, c_{3}, c_{4}$, in the embedding placed like in the first part of Figure 1.

Depending on whether $v_{i}$ appears negated or non-negated in these clauses, we differentiate between the three cases depicted in Figure 1: $v_{i}$ is literal in $c_{1}, c_{2}, c_{3}$ and $\bar{v}_{i}$ literal in 

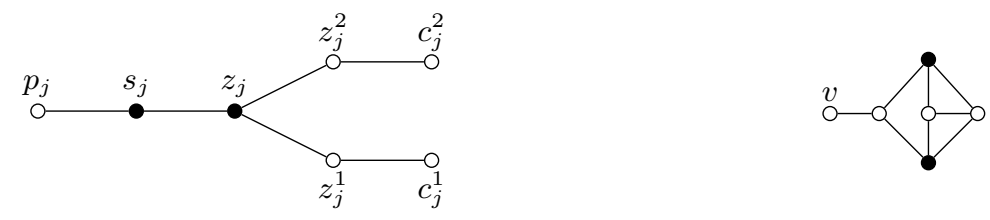

Figure 2 Construction of Theorem 10: On the left, the subgraph that replaces each clause. On the right, the subgraph that makes the graph cubic.

$c_{4} ; v_{i}$ is literal in $c_{2}, c_{4}$ and $\bar{v}_{i}$ literal in $c_{1}, c_{3} ; v_{i}$ is literal in $c_{1}, c_{2}$ and $\bar{v}_{i}$ literal in $c_{3}, c_{4}$. In Figure 1, vertices plotted in black are the ones to be put into the vertex set $S$ predetermined to be in the minimal dominating set. All other cases are rotations of the three cases of Figure 1 and/or invert the roles of $v_{i}$ and $\bar{v}_{i}$. Also, if a variable only appears positively (or negatively), it can be deleted along with the clauses which contain it. The maximum degree of the vertices which replace $v_{i}$ is three.

Replace each clause-vertex $c_{j}$ by the subgraph appearing on the left side of Figure 2 . The vertices $c_{j}^{1}, c_{j}^{2}$ somehow take the role of the old vertex $c_{j}$ regarding its neighbours: $c_{j}^{1}$ is adjacent to two of the literals of $c_{j}$ and $c_{j}^{2}$ is adjacent to the remaining literal. This way, all vertices have degree at most three and the choices of literals to connect to $c_{j}^{1}$ and $c_{j}^{2}$ can be made such that planarity is preserved.

Let $G^{\prime}$ be the graph obtained from $G$ by replacing all vertices according to the above rules. The input $G^{\prime}$ and $S:=\left\{x_{i}^{1}, x_{i}^{2}: i=1, \ldots, n\right\} \cup\left\{s_{j}, z_{j}: j=1, \ldots, m\right\}$ is a "yes"instance for Minimal Dominating Set Extension if and only if the formula associated to $G$ is a "yes"-instance for 4P3C 3 SAT.

Let $G$ be the graph associated to a satisfiable 4P3C3SAT-formula $c_{1} \wedge c_{2} \wedge \cdots \wedge c_{m}$. Consider a satisfying assignment $\phi$ for $c_{1} \wedge c_{2} \wedge \cdots \wedge c_{m}$ and the corresponding vertex-set $W:=\left\{t_{i}^{1}, t_{i}^{2}: \phi\left(v_{i}\right)=1\right\} \cup\left\{f_{i}^{1}, f_{i}^{2}: \phi\left(v_{i}\right)=0\right\}$. Let $W^{\prime}$ be an arbitrary inclusion-minimal subset of $W$ such that $\left\{c_{j}^{1}, c_{j}^{2}\right\} \cap N_{G^{\prime}}\left(W^{\prime}\right) \neq \emptyset$ for all $j \in\{1, \ldots, m\}$; $W$ itself has this domination-property since $\phi$ satisfies the formula $c_{1} \wedge c_{2} \wedge \cdots \wedge c_{m}$. By the inclusionminimality of $W^{\prime}$, the set $S \cup W^{\prime}$ is irredundant: Each vertex in $W^{\prime}$ has at least one of the $c_{j}^{k}$ as private neighbour, the vertices $x_{i}^{k}$ have either $t_{i}^{k}$ or $f_{i}^{k}$ as a private neighbour, $p n\left(s_{j}, S \cup W^{\prime}\right)=\left\{p_{j}\right\}$ and $p n\left(z_{j}, S \cup W^{\prime}\right)=\left\{z_{j}^{1}, z_{j}^{2}\right\}$. The set $S \cup W$ might however not dominate all vertices $c_{j}^{k}$. Adding the set $Y:=\left\{z_{j}^{k}: c_{j}^{k} \notin N_{G^{\prime}}(W)\right\}$ to $S \cup W$ creates a dominating set. Since for each clause $c_{j}$ either $c_{j}^{1} \in N_{G^{\prime}}\left(W^{\prime}\right)$ or $c_{j}^{2} \in N_{G^{\prime}}\left(W^{\prime}\right)$, either $z_{j}^{1}$ or $z_{j}^{2}$ remains in the private neighbourhood of $z_{j}$. Other private neighbourhoods are not affected by $Y$. At last, each vertex $z_{j}^{k} \in Y$ has the clause-vertex $c_{j}^{k}$ as private neighbour, by the definition of $Y$, so overall $S \cup W^{\prime} \cup Y$ is a minimal dominating set.

If the input $\left(G^{\prime}, S\right)$ is a "yes"-instance for Minimal Dominating Set Extension, the set $S$ can be extended to a set $S^{\prime}$ which especially dominates all vertices $c_{j}^{k}$ and has at least one private neighbour for each $z_{j}$. The latter condition implies that $S^{\prime} \cap\left\{z_{j}^{k}, c_{j}^{k}\right\}=\emptyset$ for $k=1$ or $k=2$ for each $j \in\{1, \ldots, m\}$. A vertex $c_{j}^{k}$ for which $S^{\prime} \cap\left\{z_{j}^{k}, c_{j}^{k}\right\}=\emptyset$ has to be dominated by a variable-vertex, which means that $t_{i}^{k} \in S^{\prime}\left(f_{i}^{k} \in S^{\prime}\right)$ for some variable $v_{i}$ which appears positively (negatively) in $c_{j}$. Minimality of $S^{\prime}$ requires at least one private neighbour for each $x_{i}^{k}$ which, by construction of the variable-gadgets, means that either $\left\{f_{i}^{1}, f_{i}^{2}\right\} \cap S^{\prime}=\emptyset$ or $\left\{t_{i}^{1}, t_{i}^{2}\right\} \cap S^{\prime}=\emptyset$, so each variable can only be represented either positively or negatively in $S^{\prime}$. Overall, the assignment $\phi$ with $\phi\left(v_{i}\right)=1$ if $\left\{t_{i}^{1}, t_{i}^{2}\right\} \cap S^{\prime} \neq \emptyset$ and $\phi\left(v_{i}\right)=0$ otherwise satisfies $c_{1} \wedge c_{2} \wedge \cdots \wedge c_{m}$.

Finally, $G^{\prime}$ can be transformed into a cubic planar graph, by adding the subgraph ap- 
pearing on the right side of Figure 2 once to every vertex $v$ of degree two, and twice for each degree one vertex. Add the new black vertices to the set $S$. Then all new vertices are dominated and adding another one of them to the dominating set violates irredundance. The original vertex is not dominated, adding it to the dominating set does not violate irredundance within the new vertices and the new vertices can never be private neighbours to any original vertex so the structure of $G^{\prime}$ in the above argument does not change.

\section{Approximation Algorithms}

\subsection{Bounded-degree Graphs}

Unlike the general case, UPPER DOMinAtion admits a simple constant factor approximation when restricted to graphs of maximum degree $\Delta$. This follows by the fact that any dominating set in such a graph has size at least $\frac{n}{\Delta+1}$. We show that this can be improved.

- Theorem 11. Consider some graph-class $\mathcal{G}(p, \rho)$ with the following properties:

- One can colour every $G \in \mathcal{G}(p, \rho)$ with $p$ colours in polynomial time.

- For any $G \in \mathcal{G}(p, \rho)$, MAXIMUM IndePEndent SET is $\rho$-approximable in polynomial time.

Then, for every $G \in \mathcal{G}(p, \rho)$, UPPER DOMINATION is approximable in polynomial time within ratio at most $\max \left\{\rho, \frac{\Delta \rho p+\Delta-1}{2 \rho \Delta}\right\}$.

The proof idea uses equation (2) and the fact that any maximal independent set is a minimal dominating set. We distinguish two cases, and run a different MAXIMUM INDEPENDENT SET algorithms for each case. We output the best among the computed solutions.

Any graph of maximum degree $\Delta$ can be coloured with at most $\Delta$ colours [21]; also, MAXimum IndePEndent SET is approximable within ratio $(\Delta+3) / 5$ in graphs of maximum degree $\Delta[5]$. So, the class $\mathcal{G}(\Delta,(\Delta+3) / 5)$ contains all graphs of maximum degree $\Delta$.

- Corollary 12. UPPER Domination is approximable in polynomial time within a ratio of $\left(6 \Delta^{2}+2 \Delta-3\right) / 10 \Delta$ in general graphs.

Let us note that Theorem 11 can be easily improved in the case of regular graphs. Indeed, in this case $\Gamma(G) \leqslant \frac{n}{2}$ according to [16]. Then one can conclude:

Corollary 13. UPPER DOMINATION in regular graphs is approximable in polynomial time within ratio $\Delta / 2$.

\subsection{Planar graphs}

In this section we present an EPTAS for UPPER DOMINATION on planar graphs. An EPTAS is a PTAS with running time $f\left(\frac{1}{\epsilon}\right) \cdot \operatorname{poly}(|I|)$. For this, we use techniques based on the ideas of Baker [3]. As we shall see, some complications arise in applying these techniques, because of the hardness of extending solutions to this problem.

We use the notion of outerplanar graphs. An outerplanar (or 1-outerplanar) graph $G$ is a graph such that there is a planar embedding of $G$, where all vertices are incident to the outer face of $G$. For $k>1$, graph $G$ is a $k$-outerplanar graph if there is a planar embedding of $G$, such that when all vertices, incident to the outer face are removed, $G$ is a $(k-1)$-outerplanar graph. Removing stepwise the vertices that are incident to the outer face, the vertices of $G$ can be partitioned into levels $L_{1}, \ldots, L_{k}$. We write $\left|L_{i}\right|$ for the number of vertices in level 
$L_{i}$ (if $i<1$ or $i>k$ we write $\left|L_{i}\right|=0$ ). Bodlaender [6] proved that every $k$-outerplanar graph has treewidth of at most $3 k-1$. This implies the following corollary:

- Corollary 14. The maximum minimal dominating set $\Gamma(G)$ of a k-outerplanar graph $G$ can be computed in time $f(k) n$.

To obtain the EPTAS, we split the graph $G$ into several $\ell$-outerplanar subgraphs $G_{i}$ of some small $\ell<k$. The maximum minimal dominating set $\Gamma\left(G_{i}\right)$ can be computed using the above corollary. Finally the partial solutions of $G_{i}$ are merged to obtain a minimal dominating set for $G$. In the following theorem we analyse how the maximum of the subgraphs $\Gamma\left(G_{i}\right)$ correlates to the maximum $\Gamma(G)$ of the graph $G$.

- Theorem 15. Let $G=(V, E)$ be a k-outerplanar graph with levels $L_{1}, \ldots, L_{k} \subseteq V$. For some $i \leq k$, let $G_{1}$ be the subgraph which is induced by levels $L_{1}, \ldots, L_{i-1}$ and let $G_{2}$ be the subgraph induced by levels $L_{i+1}, \ldots, L_{k}$. Then, $\Gamma\left(G_{1}\right)+\Gamma\left(G_{2}\right) \geq \Gamma(G)-\sum_{j=i-3}^{i+3}\left|L_{j}\right|$.

Proof. Given a maximum minimal dominating set for $G$ with associated partition into $(F, I, P, O)$ with $\Gamma(G)=|F|+|I|$. We construct a minimal dominating set for $G_{1}$ and $G_{2}$. We perform the following operations on a graph $G^{\prime}=\left(V^{\prime}, E^{\prime}\right)$ to remove vertices $v$ from $L_{i}$, where initially $G^{\prime}=G$. After each step we obtain a minimal dominating set for the graph induced by vertices $V^{\prime} \backslash\{v\}$ until $L_{i}$ is empty and we obtain a minimal dominating set for $G_{1}$ and $G_{2}$.

Case 1: $v \in O$.

In this case $v$ can be removed from $G$ without violating any constraint of $(F, I, P, O)$.

Case 2: $v \in P$.

Consider the partner $p n(v) \in F$ with $p n(v) \in L_{j}$ for some $i-1 \leq j \leq i+1$. In the case that there is no other $v \in G$ that is covered only by $p n(v)$, add $p n(v)$ to $O$ and remove $v$. In the case that there is a vertex $v^{\prime} \in G$ that is not covered by any other vertex except $p n(v)$, add $v^{\prime}$ to $P$ as the new neighbour of $p n(v)$ and remove $v$.

Case 3: $v \in I$ or $v \in F$.

Consider the set of vertices $W=N[v] \subseteq O \cup\{p n(v)\}(W \subseteq O$ if $v \in I)$ that are not covered by other vertices in $L_{i-1}$ or $L_{i+1}$. Hence $N[W] \subseteq P \cup O$. Our goal is to find a covering for $W$. We choose an arbitrary dominating set of the graph induced by vertices $w \in W$ to cover $W$ and we remove $v$. Covering $W$ this way might lead to conflicts as a vertex $w \in W$ is selected in the dominating set and there are edges $\left(w, w^{\prime}\right)$ for some $w^{\prime} \in P$. We solve those conflicts in the same way as in Case 2: In the case that there is no other $v \in V \backslash W$ that is covered only by $p n\left(w^{\prime}\right)$, add $p n\left(w^{\prime}\right)$ and $w^{\prime}$ to $O$. And in the case that there is a vertex $u \in V \backslash W$ that is not covered by any other vertex except $p n\left(w^{\prime}\right)$, add $u$ to $P$ as the new neighbour of $p n\left(w^{\prime}\right)$ and add $w^{\prime}$ to $O$. Note that since all vertices in $W$ belong to levels $L_{i-1}, L_{i}, L_{i+1}, w^{\prime}$ belongs to levels $L_{i-2}, \ldots, L_{i+2}$ and hence $p n\left(w^{\prime}\right)$ belongs to levels $L_{i-3}, \ldots, L_{i+3}$.

Using the above construction yields a minimal dominating set for the graph induced by vertices $V^{\prime} \backslash\{v\}$. As vertices are moved from $I$ or $F$ to $O$, the dominating set for the resulting graph is reduced. In each case we only modify the dominating set of vertices in $L_{i-3}, \ldots, L_{i+3}$ and hence the dominating set is reduced by at most $\sum_{j=i-3}^{i+3}\left|L_{j}\right|$.

Using the above theorem iteratively for several levels $L_{i_{1}}, \ldots, L_{i_{s}-1}$ yields the following.

- Corollary 16. Let $G=(V, E)$ be a k-outerplanar graph with levels $L_{1}, \ldots, L_{k} \subseteq V$. For indices $0=i_{0}<i_{1}<\ldots \leq i_{s}=k$, let $G_{j}$ be the subgraph which is induced by levels $L_{i_{j}}, \ldots, L_{i_{j+1}}$. Then, $\sum_{j=0}^{s-1} \Gamma\left(G_{j}\right) \geq \Gamma(G)-\sum_{k=0}^{s} \sum_{j=i_{k}-3}^{i_{k}+3}\left|L_{j}\right|$. 
The following algorithm shows how partial solutions of subgraphs can be used to obtain a minimal dominating set for the whole graph $G$.

- Algorithm 1. Input: A minimal dominating set of subgraphs $G_{1}=\left(V_{1}, E_{1}\right)$ and $G_{2}=$ $\left(V_{2}, E_{2}\right)$ of $G=(V, E)$, which are separated by level $L_{i}$ such that $V_{1} \cup L_{i} \cup V_{2}=V$.

1. Repeat the following steps until all vertices are covered by the dominating set.

2. Add vertex $v \in L_{i}$ which is not covered by the dominating set.

3. Remove vertices in $N[N[v]]$ from the dominating set until the dominating set is minimal.

- Theorem 17. Let $G=(V, E)$ be a k-outerplanar graph with levels $L_{1}, \ldots, L_{k} \subseteq V$. For some $i \leq k$, let $G_{1}$ be the subgraph which is induced by levels $L_{1}, \ldots, L_{i-1}$ and let $G_{2}$ be the subgraph induced by levels $L_{i+1}, \ldots, L_{k}$. Let $S_{1}$ and $S_{2}$ be a minimal dominating set of $G_{1}$ and $G_{2}$, respectively. Then Algorithm 1 returns a minimal dominating set $S$ with

$$
|S| \geq\left|S_{1}\right|+\left|S_{2}\right|-\left|L_{i-1}\right|-\left|L_{i+1}\right| .
$$

We can now state our final algorithm: An EPTAS for planar UPPER DominATion.

- Algorithm 2. Input: A k-outerplanar graph Graph $G=(V, E)$ for some $k \in \mathbb{N}$ and parameter $\epsilon$.

1. Let $\mu=\frac{36}{\epsilon}$.

2. Choose a $0 \leq x<\mu$ such that the following term is minimised

$$
\sum_{j \in \mathbb{N}}\left(\left(\sum_{i=-3}^{3}\left|L_{j \mu+x+i}\right|\right)+\left|L_{j \mu+x-1}\right|+\left|L_{j \mu+x+1}\right|\right)
$$

3. Compute $\Gamma\left(G_{i}\right)$, where $G_{i}$ is the graph induced by levels $L_{(i-1) \mu+x+1}, \ldots, L_{i \mu+x-1}$ (note that $L_{i}$ with $i<1$ or $i>k$ are empty sets).

4. Use Corollary 14 to compute the maximum minimal dominating set of $G_{i}$.

5. Use Algorithm 1 on graphs $G_{i}$ and $G_{i+1}$ with separating level $L_{i \mu+x}$ for all $0 \leq i \leq\left\lfloor\frac{k}{\mu}\right\rfloor$ to obtain a minimal dominating set for $G$.

- Theorem 18. Algorithm 2 returns a minimal dominating set $S$ of size $|S| \geq(1-\epsilon) \Gamma(G)$.

Since every $G_{i}$ is a $\mu^{\prime}$-planar graph for some $\mu^{\prime}<\mu, \Gamma\left(G_{i}\right)$ can be computed in time $f(\mu) \cdot\left|V_{i}\right|=f\left(\frac{1}{\epsilon}\right) \cdot\left|V_{i}\right|$. The time to merge the partial solutions in step 5 is bounded by $\left|V_{i}\right|^{2}$ and hence the total running time of the algorithm can be bounded by $f\left(\frac{1}{\epsilon}\right) n+O\left(n^{2}\right)$.

\section{Acknowledgements.}

We thank our colleagues David Manlove and Daniel Meister for some discussions on upper domination. Part of this research was supported by the DFG, grant FE 560/6-1.

\section{References}

1 H. AbouEisha, S. Hussain, V. V. Lozin, J. Monnot, and B. Ries. A dichotomy for upper domination in monogenic classes. In Z. Zhang, L. Wu, W. Xu, and D. Du, editors, COCOA, volume 8881 of LNCS, pages 258-267. Springer, 2014.

2 E. M. Arkin, M. A. Bender, J. S. B. Mitchell, and S. Skiena. The lazy bureaucrat scheduling problem. Inf. Comput., 184(1):129-146, 2003.

3 B. Baker. Approximation algorithms for NP-complete problems on planar graphs. J. ACM, 41:153-180, 1994. 
4 M. A. Bender, R. Clifford, and K. Tsichlas. Scheduling algorithms for procrastinators. J. Scheduling, 11(2):95-104, 2008.

5 P. Berman and T. Fujito. On the approximation properties of independent set problem in degree 3 graphs. In S. G. Akl, F. K. H. A. Dehne, J.-R. Sack, and N. Santoro, editors, $W A D S$, volume 955 of $L N C S$, pages 449-460. Springer, 1995.

6 H. L. Bodlaender. A partial $k$-arboretum of graphs with bounded treewidth. Theoretical Comput. Sci., 209:1-45, 1998.

7 N. Boria, F. D. Croce, and V. T. Paschos. On the max min vertex cover problem. In C. Kaklamanis and K. Pruhs, editors, WAOA 2013, volume 8447 of LNCS. Springer, 2014.

8 N. Bourgeois, F. D. Croce, B. Escoffier, and V. T. Paschos. Fast algorithms for min independent dominating set. Discrete Applied Mathematics, 161(4-5):558-572, 2013.

9 N. Bourgeois, B. Escoffier, and V. T. Paschos. Fast algorithms for min independent dominating set. In B. Patt-Shamir and T. Ekim, editors, SIROCCO, volume 6058 of LNCS, pages 247-261. Springer, 2010.

10 G. A. Cheston, G. Fricke, S. T. Hedetniemi, and D. P. Jacobs. On the computational complexity of upper fractional domination. Discrete Applied Mathematics, 27(3):195-207, 1990.

11 E. J. Cockayne, O. Favaron, C. Payan, and A. G. Thomason. Contributions to the theory of domination, independence and irredundance in graphs. Discrete Mathematics, 33(3):249258, 1981.

12 B. Courcelle. The monadic second-order logic of graphs. I. Recognizable sets of finite graphs. Inf. Comput., 85:12-75, 1990.

13 M. M. Halldórsson. Approximating the minimum maximal independence number. Inf. Process. Lett., 46(4):169-172, 1993.

14 E. O. Hare, S. T. Hedetniemi, R. C. Laskar, K. Peters, and T. Wimer. Linear-time computability of combinatorial problems on generalized-series-parallel graphs. In D. S. Johnson et al., editors, Discrete Algorithms and Complexity (Academic Press NY), 1987.

15 T. W. Haynes, S. T. Hedetniemi, and P. J. Slater. Fundamentals of Domination in Graphs, volume 208 of Monographs and Textbooks in Pure and Applied Mathematics. Marcel Dekker, 1998.

16 M. A. Henning and P. J. Slater. Inequalities relating domination parameters in cubic graphs. Discrete Mathematics, 158(1-3):87-98, 1996.

17 J. Håstad. Clique is hard to approximate within $n^{1-\epsilon}$. Acta Mathematica, 182:105-142, 1999.

18 J. Hurink and T. Nieberg. Approximating minimum independent dominating sets in wireless networks. Inf. Process. Lett., 109(2):155-160, 2008.

19 M. S. Jacobson and K. Peters. Chordal graphs and upper irredundance, upper domination and independence. Discrete Mathematics, 86(1-3):59-69, 1990.

20 J. Kratochvíl. A special planar satisfiability problem and a consequence of its NPcompleteness. Discrete Applied Mathematics, 52:233-252, 1994.

21 L. Lovász. Three short proofs in graph theory. Journal of Combinatorial Theory Series B, 19:269-271, 1975.

22 C. H. Papadimitriou and M. Yannakakis. Optimization, approximation, and complexity classes. J. Comput. Syst. Sci., 43(3):425-440, 1991.

23 M. Zehavi. Maximum minimal vertex cover parameterized by vertex cover. In G. F. Italiano, G. Pighizzini, and D. Sannella, editors, MFCS 2015, volume 9235 of Lecture Notes in Computer Science, pages 589-600. Springer, 2015. 


\section{A Omitted Proofs}

\section{A.1 Proof of Lemma 1}

Proof. Let $G$ be a connected graph with $n>0$ vertices and let $D$ be an upper dominating set with an associated partition $(F, I, P, O)$ as defined above.

We first show that if $\Gamma(G)>\alpha(G)$ then $|F| \geq 2$ (in fact, one can show that then $|F| \geq 3$ but that is not necessary for our proof). Indeed, if $|F|=0$, then the upper dominating set is also an independent set, and thus $\Gamma(G)=\alpha(G)$, and according to our definition of partition $(F, I, P, O)$, we have $|F| \neq 1$ (see Property 1 of this partition).

Now, if $|F| \geq 2$ then the subgraph of $G$ induced by $F \cup P$ contains an independent set of size 2 consisting of a vertex in $F$, say $v$, and a vertex in $P$, say $u$, such that $v$ and $u$ are not adjacent. Since in the original graph $G$, there are no edges between the vertices in $I$ and the vertices in $F \cup P$ (Property 4 ), $I \cup\{u, v\}$ forms an independent set of size $|I|+2$. This sets a lower bound on the independence number and we have $\alpha(G) \geq|I|+2$, that is, $|I| \leq \alpha(G)-2$.

From the above, it follows that if $\Gamma(G)>\alpha(G)$ then $|I| \leq \alpha(G)-2$.

\section{A.2 Proof of Lemma 2}

Proof. We consider a graph $G$ with $n>0$ vertices and an upper dominating set $D$ with an associated partition $(F, I, P, O)$ as defined above. The left inequality comes from the fact that any maximal independent set is a minimal dominating set. For the right inequality, we examine separately the following two cases.

1. $\Gamma(G)=\alpha(G)$. Then we trivially have $\Gamma(G) \leq \alpha(G)$.

2. $\Gamma(G)>\alpha(G)$.

From the fact that $|F|=|P|$ (from Property 3) we have $|F|=\frac{n-|I|-|O|}{2} \leq\left\lfloor\frac{n-|I|}{2}\right\rfloor$ and thus

$$
\Gamma(G)=|F|+|I| \leq\left\lfloor\frac{n+|I|}{2}\right\rfloor
$$

From the above and Lemma 1 we have

$$
\Gamma(G) \leq\left\lfloor\frac{n+|I|}{2}\right\rfloor \leq\left\lfloor\frac{n+\alpha(G)-2}{2}\right\rfloor \leq \frac{n}{2}+\frac{\alpha(G)}{2}-1
$$

This concludes the proof of the claim.

\section{A.3 Proof of Lemma 3}

Proof. Let $G$ be a connected graph with $n>0$ vertices, maximum degree $\Delta$ and an upper dominating set $D$ with an associated partition $(F, I, P, O)$ as defined above. Our argument is similar to the one in Lemma 2: The left inequality comes from the fact that any maximal independent set is a minimal dominating set. For the right inequality, we examine separately the following two cases.

1. $\Gamma(G)=\alpha(G)$. Then we trivially have $\Gamma(G) \leq \alpha(G)$.

2. $\Gamma(G)>\alpha(G)$. Again, we obtain:

$$
\Gamma(G)=|F|+|I|=\frac{n+|I|-|O|}{2}
$$

We next derive an improved lower bound on $|O|$. Let $e$ be the number of edges adjacent with vertices from $I$. As $G$ is of minimum degree $\delta$, we have $e \geq \delta|I|$. As the vertices in 
$I$ are only adjacent with the vertices in $O$, there are at least $e$ edges that have exactly one end vertex in $O$. Since $G$ has maximum degree $\Delta$, we have that $|O| \geq\left\lceil\frac{e}{\Delta}\right\rceil \geq\left\lceil\frac{\delta|I|}{\Delta}\right\rceil$. From the above and Lemma 1 we have

$$
\begin{aligned}
\Gamma(G) & \leq\left\lfloor\frac{n+|I|-\left\lceil\frac{\delta \mid I I}{\Delta}\right\rceil}{2}\right\rfloor \leq \frac{n+|I|-\frac{\delta|I|}{\Delta}}{2}=\frac{n+\frac{(\Delta-\delta)|I|}{\Delta}}{2} \\
& \leq \frac{n+\frac{(\Delta-\delta)}{\Delta}(\alpha(G)-2)}{2}=\frac{n}{2}+\frac{\Delta-\delta}{2 \Delta} \alpha(G)-\frac{\Delta-\delta}{\Delta}
\end{aligned}
$$

To combine these two upper bounds we take their maximum, which concludes the proof.

\section{A.4 Proof of Corollary 5}

Proof. Assume there were, for some $\varepsilon>0$, a factor- $n^{1-\varepsilon}$ approximation algorithm $A$ for Maximum Minimal Hitting Set. Then, choose $d$ such that $1 / d \leq \varepsilon / 2$ and hence $(d-$ $1) / d \geq 1-\varepsilon / 2$. Then, $A$ would be a factor- $n^{(d-1) / d-\varepsilon / 2}$ approximation algorithm when restricted to hypergraphs with hyperedges of size at most $d$, contradicting Theorem 4.

\section{A.5 Proof of Theorem 6}

Proof. We construct an approximation-preserving reduction from Maximum Minimal Hitting Set. Given a hypergraph $G=(V, F)$ as an instance of Maximum Minimal Hitting Set, we define a graph $G^{\prime}=\left(V^{\prime}, E^{\prime}\right)$ as an instance of Upper Domination as follows: $V^{\prime}$ contains a vertex $v_{i}$ associated to any vertex $i$ from $V$, a vertex $u_{e}$ for any edge $e \in F$ and a new vertex $v$. $E^{\prime}$ contains edges such that $G^{\prime}[V]$ and $G^{\prime}[F]$ are cliques. Moreover, $v$ is adjacent to every vertex $v_{i} \in V$, and $\left(v_{i}, u_{e}\right) \in E^{\prime}$ if and only if $i \in e$ in $G$.

First we show that given a solution $S$ that is a minimal hitting set in $G, S$ is also a minimal dominating set in $G^{\prime}$. Indeed if $S$ is a hitting set in $G$ then $S$ is a dominating set in $G^{\prime}$. If $S$ is minimal, that is, any proper subset $S^{\prime} \subset S$ is no longer a hitting set, then it is also the case that $S^{\prime}$ is no longer a dominating set in $G^{\prime}$. That implies that $\operatorname{opt}\left(G^{\prime}\right) \geq \operatorname{opt}(G)$.

Consider now an upper dominating set $S$ for $G^{\prime}$. To dominate the vertex $v, S$ has to contain at least one vertex $w \in V \cup\{v\}$. If $S$ contains one vertex $u_{e} \in E$, then the set $\left\{w, u_{e}\right\}$ is already dominating. If we want a solution of cardinality more than two, then $S \subseteq V$. If $S \subseteq V$ is a minimal dominating set in $G^{\prime}, S$ is also a minimal hitting set in $G$ since $S$ covers all hyperedges in $G$ if and only if it dominates all edge-vertices in $G^{\prime}$. So starting with any minimal dominating set $S$ of $G^{\prime}$ of cardinality larger than two, $S$ is also a minimal hitting set of $G$. The result now follows from Corollary 5 .

\section{A.6 Proof of Theorem 7}

Proof. The proof is by induction on $d$. For $d=1$, if every vertex appears in a hyperedge, any hitting set must contain all vertices, so we are done. For $d>1$, we do the following: first, greedily construct a maximal set $M \subseteq F$ of pair-wise disjoint hyperedges. If $|M| \geq n^{1 / d}$ then we know that any hitting set of $G$ must contain at least $n^{1 / d}$ vertices. So, we simply produce an arbitrary feasible solution by starting with $V$ and deleting redundant vertices until our hitting set becomes minimal.

Suppose then that $|M|<n^{1 / d}$. Let $H$ be the set of all vertices contained in $M$, so $|H|<d|M|=O\left(n^{1 / d}\right)$. Clearly, $H$ is a hitting set of $G$ (otherwise $M$ is not maximal), but it is not necessarily minimal. Let us now consider all sets $S \subseteq H$ with the following two properties: $|S| \leq d-1$ and all edges $e \in F$ have an element in $V \backslash S$ (in other words, $V \backslash S$ is 
a hitting set of $G$ ). For such a set $S$ and a vertex $u \in V \backslash H$ we will say that $u$ is seen by $S$, and write $u \in B(S)$, if there exists $e \in F$ such that $e \cap H=S$ and $u \in e$. Intuitively, what we are trying to do here is find a set $S$ that we will not place in our hitting set. Vertices seen by $S$ are then vertices which are more likely to be placeable in a minimal hitting set.

Let $B_{i}$ be the union of all $B(S)$ for sets $S$ with $|S|=i$. Since every vertex appears in a hyperedge, all vertices of $V \backslash H$ are seen by a set $S$, and therefore belong in some $B_{i}$. Therefore, the union of all $B_{i}$ has size at least $|V \backslash H| \geq n-n^{1 / d}=\Omega(n)$. The largest of these sets, then, has size at least $\frac{n-n^{1 / d}}{d}=\Omega(n)$. Consider then the largest such set, which corresponds to sets $S$ with size $s$. There are at most $\left(\begin{array}{c}|H| \\ s\end{array}\right)=O\left(n^{s / d}\right)$ such sets $S$. Since all together they see $\Omega(n)$ vertices of $V \backslash H$, one of them must see at least $\Omega\left(n^{1-\frac{s}{d}}\right)$ vertices. Call this set $S_{m}$. Consider now the hypergraph induced by $S_{m} \cup B\left(S_{m}\right)$. If we delete the vertices of $S_{m}$ from this hypergraph, we get a hypergraph where every hyperedge has at most $d-s$ vertices. By induction, we can in polynomial time find a minimal hitting set of this hypergraph with at least $\Omega\left(\left(n^{1-\frac{s}{d}}\right)^{\frac{1}{d-s}}\right)=\Omega\left(n^{1 / d}\right)$ vertices. Call this set $H^{\prime}$.

We are now ready to build our solution. Start with the set $V \backslash\left(S_{m} \cup B\left(S_{m}\right)\right)$ and add to it the vertices of $H^{\prime}$. First, this is a hitting set, because any hyperedge not hit by $V \backslash\left(S_{m} \cup B\left(S_{m}\right)\right)$ is induced by $S_{m} \cup B\left(S_{m}\right)$, and $H^{\prime}$ hits all such hyperedges. We now proceed to make this set minimal by arbitrarily deleting redundant vertices. The crucial point here is that no vertex of $H^{\prime}$ is deleted, since this would contradict the minimality of $H^{\prime}$ as a hitting set of the hypergraph induced by $S_{m} \cup B\left(S_{m}\right)$. Thus, the resulting solution has size $\Omega\left(n^{1 / d}\right)$.

\section{A.7 Proof of Theorem 8}

Proof. We present a reduction from Maximum IndePendent SET on cubic graphs, which is known to be APX-hard [22]. Let $G=(V, E)$ be the cubic input graph for MAXIMUM IndePEndent Set. Construct a cubic graph $G^{\prime}$ from $G$ by replacing every edge $(u, v) \in E$ by the following construction introducing six new vertices for each edge:

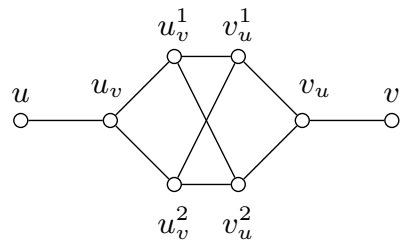

If $I S$ is an independent set for $G$, the corresponding vertex-set in $G^{\prime}$ can be extended to an upper dominating set $S$ of cardinality at least $|I S|+3|E|$ in the following way: For every edge $(u, v)$ with $v \notin I S$ add $\left\{v_{u}, u_{v}^{1}, u_{v}^{2}\right\}$ to $S$ :

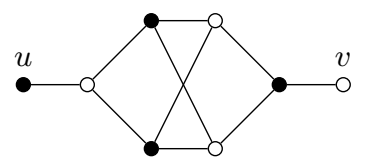

Since $I S$ is independent, this procedure chooses three vertices for each edge-gadget in $G^{\prime}$ and creates an independent set $S$ of cardinality $|I S|+3 \mathrm{~m}$. If there was some vertex not dominated by $S$ we could add it to $S$ without violating independence and $S$ will only increase in cardinality. Finally we arrive at a maximal independent and consequently minimal dominating set of cardinality at least $|I S|+3|E|$. 
Let $S$ be an upper dominating set for $G^{\prime}$. Minimality yields that for every edge $(u, v) \in E$ at most three of the vertices added for this edge can be in $S$. Consider an original edge $(u, v) \in E$ such that $u, v \in S$. From the vertices added for this edge, only two can be in $S$ : Observe that any two out of $\left\{u_{v}^{1}, u_{v}^{2}, v_{u}^{1}, v_{u}^{2}\right\}$ already dominate the whole subgraph. The set $\left\{u_{v}, v_{u}\right\}$ is also already dominating so there is no minimal dominating set of cardinality three in this case.

Consider the set $S^{\prime}=S \cap V$ as potentially independent set for the original graph $G$. If there are two vertices $u, v \in S^{\prime}$ such that $(u, v) \in E$, the corresponding edge-gadget only adds two vertices to $S$. By successively deleting vertices from $S^{\prime}$ as long as there is a conflict with respect to independence, we arrive at an independent set of cardinality at least $|S|-3|E|$. APX-hardness now follows by observing that, since $G$ is cubic we have $\alpha(G)=\Theta(|V|)$ and $|E|=\Theta(|V|)$.

\section{A.8 Proof of Theorem 9}

Proof. We would like to use the same proof as in Theorem 8, starting from subcubic MAXIMUM INDEPENDENT SET on planar graphs, which is known to be NP-hard. Unfortunately, the gadget we used destroys planarity. We therefore use a slightly modified version: we remove the edges $\left(u_{v}^{1}, v_{u}^{2}\right)$ and $\left(v_{u}^{1}, u_{v}^{2}\right)$. Note that the reduction now produces a subcubic planar graph, since some vertices have degree two.

The forward direction of the reduction is identical: if there exists an independent set of size $|I S|$ in $G$, we construct the same minimal dominating set of size $|I S|+3|E|$. For the converse direction, it is not hard to see that it is still impossible to select four internal vertices of the edge gadget while maintaining minimality. The only difference with the previous proof is that we need to argue again that if a minimal dominating set contains both $u$ and $v$, it may only contain up to two internal vertices. Suppose then that $u, v \in S$ for some edge $(u, v) \in E$. First, observe that it cannot be the case that $u_{v}^{1}, v_{u}^{1} \in S$, because then $u_{v}^{1}$ has no private neighbour. So, out of the two "top" vertices, $S$ may select at most one, and similarly for the "bottom" vertices. If $u_{v}, v_{u} \notin S$ we are done, as $S$ selects at most two internal vertices from the gadget. If on the other hand we assume without loss of generality that $u_{v} \in S$, then one of $u_{v}^{1}, u_{v}^{2}$ must be the private neighbour of $u_{v}$. If (again without loss of generality) it is $u_{v}^{1}$ this means that $v_{u}^{1} \notin S$. It is not hard to see now that out of $\left\{u_{v}^{2}, v_{u}^{2}, v_{u}\right\}$ at most one more vertex can be added to $S$ while maintaining minimality.

\section{A.9 Proof of Theorem 11}

Proof. The approximation algorithm consists of running two independent set algorithms, by greedily augmenting solutions computed in order to become maximal for inclusion and of returning the best among them, denoted by $U$. Recall that any maximal (for inclusion) independent set is a feasible upper dominating set.

The algorithms used are:

(i) the $\rho$-approximation algorithm assumed for $\mathcal{G}(p, \rho)$ and

(ii) the (also assumed) algorithm that colours the vertices of the input graph with $p$ colours and takes the largest colour as solution for UPPER DOMINATION.

Revisit (2). If the maximum there, is realised by the first term, then we are done since the $\rho$-approximation MAXimum InDEPEndent SET-algorithm assumed, also achieves ratio $\rho$ for UPPER DOMINATION.

Suppose now that the maximum in (2) is realised by the second term and set $\alpha(G)=n / t$, for some $t \geqslant 1$ that will be fixed later. In order to simplify calculations we will use the 
following bounds for $\Gamma(G)$, easily derived from 2 :

$$
\begin{aligned}
\Gamma(G) & \leqslant \frac{n}{2}+\frac{\alpha(G)(\Delta-1)}{2 \Delta}=\frac{\Delta(t+1)-1}{2 t \Delta} n \\
\Gamma(G) & \leqslant \frac{\Delta(t+1)-1}{2 \Delta} \alpha(G)
\end{aligned}
$$

Expression (4) leads to:

$$
\alpha(G) \geqslant \frac{2 \Delta}{\Delta(t+1)-1} \Gamma(G)
$$

If the solution $U$ returned by the algorithm is the one of item (i), Equation (5) yields:

$$
\begin{aligned}
|U| & \geqslant \rho \alpha(G) \geqslant \frac{2 \rho \Delta}{\Delta(t+1)-1} \Gamma(G) \\
\frac{\Gamma(G)}{|U|} & \leqslant \frac{\Delta(t+1)-1}{2 \rho \Delta}
\end{aligned}
$$

Assume now that the UPPER Domination-solution returned is the one by item (ii) and note that the largest colour computed is assigned to at least $n / p$ vertices of the input graph which, obviously, form an independent set. So, in this case, $|U| \geqslant n / p$ and using (3), the ratio achieved is:

$$
\frac{\Gamma(G)}{|U|} \leqslant \frac{\frac{\Delta(t+1)-1}{2 t \Delta} n}{\frac{n}{p}}=\frac{p(\Delta(t+1)-1)}{2 t \Delta}
$$

Equality of ratios in (6) and (7) implies $t=\rho p$ and setting it to either one of those leads to the second term of the max expression in the equation of Theorem 11.

\section{A.10 Proof of Corollary 14}

Proof. It is not hard to see that UPPer Domination is expressible in MSO logic, therefore fixed-parameter tractability for treewidth follows directly from known meta-theorems [12].

\section{A.11 Proof of Theorem 17}

Proof. By definition of the algorithm, vertices $v \in L_{i}$ that are not covered by vertices in $L_{i-1}$ or $L_{i+1}$ are added to the dominating set. This leads to a violation of the minimality of the dominating set if there is a vertex $w \in N[v] \cap P$ such that there is no other vertex that is covered only by $p n(w) \in F$. In that case, move $w$ and $p n(w)$ to $O$.

Since we remove for every vertex $w \in N[v]$ in $L_{i-1}$ or $L_{i+1}$ at most one vertex from the dominating set, at most $\left|L_{i-1}\right|+\left|L_{i+1}\right|$ vertices are removed from the dominating set by Algorithm 1.

\section{A.12 Proof of Theorem 18}

Proof. By definition of the algorithm, vertices $v \in L_{i}$ that are not covered by vertices in $L_{i-1}$ or $L_{i+1}$ are added to the dominating set. This leads to a violation of the minimality of the dominating set if there is a vertex $w \in N[v] \cap P$ such that there is no other vertex that is covered only by $p n(w) \in F$. In that case, move $w$ and $p n(w)$ to $O$.

Since we remove for every vertex $w \in N[v]$ in $L_{i-1}$ or $L_{i+1}$ at most one vertex from the dominating set, at most $\left|L_{i-1}\right|+\left|L_{i+1}\right|$ vertices are removed from the dominating set by Algorithm 1. 